\title{
Performance Improvement in MIMO-OFDM Systems based on adaptive Whale Elephant Herd Optimization algorithm
}

\author{
Kurra. Upendra Chowdary, B. Prabhakara Rao
}

\begin{abstract}
Multiple-input, multiple-output orthogonal frequency-division multiplexing (MIMO-OFDM) is the leading air interface for $4 G$ and $5 G$ broadband wireless communications. Cognitive Radio (CR) is the trending technology, which facilitates spectrum sensing to determine the inadequate bands in the spectrum. Though there are so many spectrum sensing techniques, the existing methods suffered a lot in the communication environment. In the traditional CR network, data transmission is constrained to the secondary user within the available bands. With the aim to increase spectrum efficiency and throughput, this paper proposed the hybrid mixture model using Adaptive Whale Elephant Herd Optimization (Adaptive WEHO) algorithm for spectrum sensing. Adaptive WEHO is the integration of Elephant-Herd Optimization (EHO), and Whale Optimization Algorithm (WOA), with the adaptive concept. The signal received from the OFDM antenna is used to analyze the availability of spectrum based on the signal energy and Eigen statistics. The CR searches the availability of channel and makes the connection when it determines a free channel. Here, the spectrum sensing is achieved by the Gaussian Mixture Model (GMM), which is trained by the proposed Adaptive WEHO algorithm. The proposed Adaptive WEHO algorithm uses the foraging behavior of whales and the herding behavior of elephants, which is applied in the spectrum sensing technique to perform the optimal sensing. The proposed Adaptive WEHO attained better performance with the metrics of probability of detection as 1.0238, and the probability of false alarm as 0.01075 , respectively. The proposed method ensures the effective communication in CR without any interference.
\end{abstract}

Keywords: Cognitive Radio (CR), spectrum sensing, Gaussian Mixture Model (GMM), MIMO, hybrid optimization.

\section{INTRODUCTION}

In the recent decades, the wireless environment skilled an enormous development to enhance the spectrum usage in the communication model termed as Cognitive radio (CR) [1] [7] [8]. CR is the rising technology used to enhance the spectrum efficiency in data transmission. The CR system requires an accurate and efficient technique of spectrum sensing to eliminate the cause of interference to the primary user in order to enhance the QoS [4].

Revised Manuscript Received on October 30, 2019.

* Correspondence Author

Ravindra Parab*, School of Mechatronics, Symbiosis University of Applied Sciences, Indore, India.

Smita Prajapati, School of Mechatronics, Symbiosis University of Applied Sciences, Indore, India.

(c) The Authors. Published by Blue Eyes Intelligence Engineering and Sciences Publication (BEIESP). This is an open access article under the CC BY-NC-ND license (http://creativecommons.org/licenses/by-nc$\underline{\mathrm{nd} / 4.0 /)}$
CR system consists of two categories of users, like primary user and the secondary user. Here, the primary user is the licensed one, who has individual influence in the part of band. The secondary user is referred as unlicensed user, who utilizes the CR for accessing the spectrum while the spectrum is not used by the primary one. The secondary user is freely permits to access the spectrum in data transmission when the Quality of Service (QoS) of the primary user is not lower than the threshold value [2]. This feature makes the user to continuously access the spectrum for finding the transmission opportunities. In general, the primary user suggest the interference constraint factor to the secondary user, hence the secondary users are enclosed to forward the data under the constraint factors [2]. CR allows the secondary user to access the frequency spectrum wile it is not used by the primary user [1] [8] [9].

To increase the efficiency of spectrum sensing, the Multiple Input Multiple Output (MIMO) technique is used in the CR network [2]. Spectrum sensing is a technique used to find the available spectrum and to eliminate the interference based on the primary user in order to increase the utilization of spectrum [1] [10] [11]. In the wireless scenario, to achieve the perfect sensing results a challenging issue. Due to the uncertainty of wireless channel, interference, quantization error, and feedback delay the sensing error occurs in the wireless channel [1] [12] [13]. CR is implemented in the heterogeneous network named as HCRN, which helps to increase the spectrum efficiency in network [1]. Spectrum sensing is a part of CR network and several research works are performed based on the sensing technique of CR [2]. Some of the spectrum sensing methods are: generalized likelihood ratio test (GLRT), detection uisng Eigen values of covariance matrix, detection using the correlation structure of OFDM signals [15], and Energy detection [14], respectively [2]. Some other spectrum sensing models are: matched filter model, and cyclo-stationary detection mechanism [4]. The cyclostationary model achieves better spectrum sensing, but the computational complexity is too high. The problem of noise power is solved using the noise power elimination method, which achieves better detection performance [4].

The primary intention of this research is to develop a hybrid mixture model using the proposed optimization algorithm. The OFDM signals are received from the antenna and the test statistics are computed from the signals based on Eigen statistics and signal energy. 
The resulted signal is passed to the GMM, which effectively performs the spectrum sensing by maximizing the throughput and spectral efficiency. The major contribution of this research is explained as follows:

- The training procedure of GMM is done with the proposed Adaptive WEHO approach. By training the Gaussian parameters, the proposed hybrid mixture model revealed enhanced throughput and efficiency.

The rest of the paper is organized as follows: Section 2 elaborates the review of various existing spectrum sensing methods. Section 3 demonstrates the proposed hybrid mixture model, and section 4 elaborates the results and discussion of the proposed optimization algorithm. Finally, section 5 concludes the paper.

\section{MOTIVATION}

In this section, some of the existing spectrum sensing methods are reviewed along with their merits and demerits.

\section{A. Literature survey}

Various existing spectrum methods are reviewed in this section. Thangaraj, C.A. and Aruna, T [1] introduced an energy efficient power allocation (EEPA) algorithm to enhance the energy efficiency of heterogeneous CRN. It effectively guaranteed the QoS under imperfect sensing and offered better data rate and energy efficiency. However, it failed to perform the power allocation scheme with spectrum sensing in femto cell network. Viswanath, $\mathrm{V}$ et al. [2] introduced an interweave approach in MIMO cognitive radio for spectrum access using primary and secondary user. It used the poison process to model the activities of primary user. However, the interweave approach provided better performance for the probability of alarm in the MIMO channel. It failed to provide closed form expression with the interference on secondary user. Patel, A et al. [3] introduced an uncertainty-aware robust detector to solve the issues of spectrum sensing in CRN using multiple transmit and receive antennas. Here, the performance of the detector was revealed using the closed form expression. It yielded better performance in the SNR regime. This model generates the decision rule at fusion center using local decision. However, it failed to integrate the censoring of local decision with the fusion center. Ren, X. and Chen, $\mathrm{C}$ [4] developed a sample variance detection algorithm (SVD) in MIMO system. It used the row vector of covariance matrix as samples and generated the detection statistics based on sample variance. With the alarm probability, the judgment threshold was derived. It effectively reduced the sampling points and increased the ability of detection in MIMO system. It failed to minimize the interference in secondary user.

\section{B. Challenges}

Some of the challenges associated with the existing spectrum sensing methods are discussed below:

- Due to the insufficiency of licensed bands, the existing price of the spectrum is too high, which resulted in a major challenge in spectrum congestion [2].

- It is required to find the presence of primary user by the secondary user in the licensed spectrum and need quit the frequency band to avoid the interference, which poses a major challenge in spectrum sensing [10].

- To detect the weak signals in the noisy channel environment with less detection probability poses a key challenge in spectrum sensing.

\section{PROPOSED HYBRID MIXTURE MODEL FOR SPECTRUM SENSING IN MIMO SYSTEMS}

In this paper, the spectrum sensing is performed using the proposed Adaptive WEHO algorithm. At first, the signals are received from OFDM system and are analyzed with the Eigen statistics and energy detector for deriving the energy using hypothesis test. The Eigen statistics and the energy of the signal are passed to the GMM classifier for deriving the spectrum availability to achieve effective communication. The GMM classifier is trained by the proposed Adaptive WEHO algorithm, which is the integration of Whale Optimization Algorithm (WOA) [5], and Elephant Herd Optimization (EHO) [6], respectively. Figure 1 shows the block diagram of the proposed hybrid mixture model.

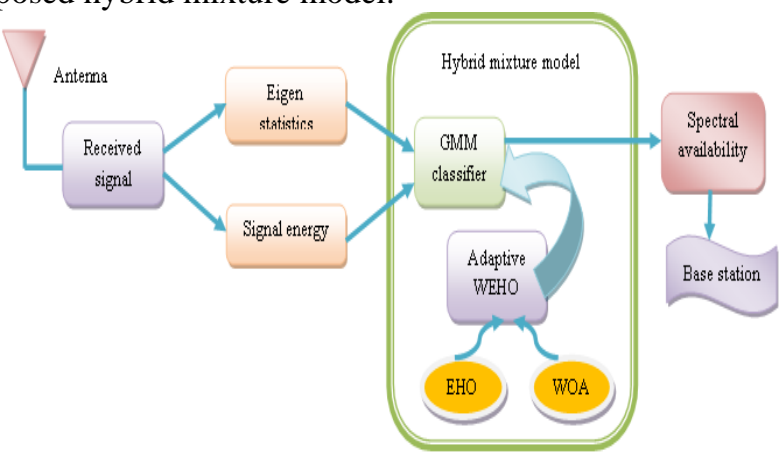

Figure 1. Proposed hybrid mixture model for spectrum sensing

\section{A. Generating test statistics for spectrum sensing}

The test statistics used to analyze the availability of channel in CR network is elaborated in this section. Let us consider the CR network environment with $p$ number of transmitters and $q$ number of receivers, which are termed as sensors. The data transmission between the transmitters and the receivers are done using the radio channel. To achieve the communication process, it is necessary to ensure that there must be any free channel in CR. The CR searches the availability of channel and makes the connection when it determines a free channel. CR effectively determines the unoccupied and occupied channel, and the data samples send by the transmitter is received by the sensors $q$. Hence, in this research, a hybrid model is proposed to find the availability of channel efficiently. The hybrid model detects the availability of channel using Eigen statistics and signal energy. However, the data matrix $M$ generated based on the data samples is represented as,

Published By: 


$$
M=\left[M_{m n}\right]_{q \times v} \quad ;(1 \leq m \leq q) ;(1 \leq n \leq v)
$$

where, $M_{m n}$ indicates $n^{\text {th }}$ sample received using $m^{\text {th }}$ sensor, and $v$ denotes the number of data samples. The transmitted signal matrix is represented as,

$$
N=\left[N_{a n}\right]_{p \times v}
$$

where, $p$ denotes the number of transmitters, $N_{a n}$ represents the signal received from $a^{\text {th }}$ transmitter, and $v$ denotes total data samples. The communication takes place through the radio channel with the unoccupied band. The communication performed using the OFDM system partition the data stream into narrow band channel for sharing the bandwidth availability. The OFDM present in the data channel maximizes the utilization of spectrum energy by reducing the interference. The input signal used here is either phase or amplitude-modulated, and the signal is changed from serial to parallel. The modulated signal is carried by the OFDM carrier and the complex signal is generated using Inverse Fast Fourier Transform (IFFT). Then, the data stream is converted into serial stream based on the parallel to serial converter. However, the data matrix is represented as,

$$
M=[A * B]+L
$$

where, $L$ and $A$ are thermal noise and channel gain matrix. However, the channel gain matrix is denoted as,

$$
A=\left[A_{m a}\right]_{q \times p}
$$

where, $A_{m a}$ denotes the channel gain between $m^{\text {th }}$ sensor and $a^{\text {th }}$ transmitter. The signal that are transmitted in the CR network is affected by the thermal noise such that the thermal noise matrix is represented as,

$$
D=\left[D_{m n}\right]_{q \times v}
$$

where, $D_{m n}$ denotes thermal noise. Here, the data sample matrix is used to calculate the Eigen statistics and signal energy, which are fed as input to the hybrid model for predicting the availability of channel. The covariance matrix is calculated as,

$$
I=\operatorname{Expected}\left[M M^{+}\right]
$$

where, Expected [ ]denotes expected value, and $M^{+}$ denotes the conjugate of $M$. With the covariance matrix, the signal energy and Eigen statistics are passed as input to the hybrid mixture model. However, the Eigen values of $I$ is represented as,

$$
\left\{\lambda_{1} \geq \lambda_{2} \geq \lambda_{q}\right\}
$$

Here, the energy statistics is defined as,

$$
\lambda=\frac{\lambda_{1}}{\frac{1}{q} \sum_{m=1}^{q} \lambda_{m}}
$$

where, $\lambda_{m}$ denotes Eigen value of $m^{\text {th }}$ sensor, and the Eigen statistics is represented as,

$$
H=\frac{1}{p \times v^{2}} \sum_{m=1}^{q} \lambda_{m}
$$

where, $v^{2}$ represents thermal noise factor.

\section{B. Gaussian Mixture Model based on unsupervised learning}

In the hybrid mixture model, the availability of spectrum is predicted with the GMM classifier and Adaptive WEHO algorithm, which optimally tunes the GMM parameters to achieve better prediction performance. At first, the Eigen statistics and the signal energy are given as input to the GMM [16] for generating the class. The output obtained from the GMM is indicated as,

$$
O=G M M[\lambda, H]
$$

where, $O$ indicates the predicted output that determines the class as either un-occupied or occupied. The occupied bands are not assigned, while the un-occupied bands are assigned to the user for achieving the communication. GMM effectively detects the un-occupied and the occupied bands using the Eigen statistics and signal energy based on the probability of class. Let us consider the intermediate class as $C=\left\{C_{x_{1}}, \ldots C_{x_{r}}\right\}$ and $r$ denotes the number of class. Here, the class is defined using the class variable $x=\left[x_{1}, x_{2} \ldots, x_{r}\right]$. Thus, the GMM is represented as,

$$
P(y)=\sum_{x} P(y, x)=\sum_{x} P(x) P(y / x)=\sum_{l=1}^{r} P\left(x_{l}\right) P\left(y / x_{l}\right)=\sum_{l=1}^{r} \alpha_{l} \times Z\left(y / \beta_{l}, \sum_{l}\right)
$$

where, $x$ denotes the vector, and $x_{l}$ indicates the condition at which $x_{l}=1$ and $x_{g}=0$ for $g \neq l$. $r$ denotes the classes, and $x$ represents the class value. Here, $\alpha_{l}$ denotes mixing coefficient, $\beta$ denotes mean, and $\sum_{l}$ is the covariance. However, the assumption of GMM is represented as,

$$
P\left(x_{l}\right)=\alpha_{l} ; P\left(y / x_{l}\right)=Z\left(y / \beta_{l}, \sum_{l}\right)
$$

Here, $P\left(x_{l}\right)$ denotes prior probabilities, and $P\left(y / x_{l}\right)$ represents likelihood. Moreover, the posterior probability of class is represented as,

$$
\operatorname{post}\left(x_{l} / y\right)=\frac{P\left(x_{l}\right) P\left(y / x_{l}\right)}{P(y)}=\frac{\alpha_{l} Z\left(y / \beta_{l}, \sum_{l}\right)}{\sum_{l=1}^{r} \alpha_{l} \times Z\left(y / \beta_{l}, \sum_{l}\right)}
$$

where, $P\left(x_{l} / y\right)$ denotes class posterior probability, $P\left(x_{l}\right)$ denotes prior probabilities, and $P\left(y / x_{l}\right)$ represents likelihood. The Gaussian parameters of GMM classifier is trained by the proposed Adaptive WEHO optimization algorithm.

\section{Proposed Adaptive WEHO algorithm for spectrum sensing}

The proposed Adaptive WEHO algorithm is used to train the GMM in order to ensure the effectiveness of spectrum sensing. However, the proposed Adaptive WEHO is the integration of EHO [5], and WOA [6], with the adaptive concept. The proposed optimization algorithm operates with the hunting behavior of humpback whales. 
The most interesting fact in the Adaptive WEHO algorithm is the encircling and the foraging behavior of the whales to search the location of prey in search space. The hunting mechanism of the whales is processed using three phases, such as searching, attacking, and encircling the prey. The detail description of spectrum sensing using the adaptive WEHO is given below with the algorithmic steps.

\section{a) Solution encoding}

It is the representation of solution, and the dimension of the solution is equivalent to the dimension of Gaussian parameters, which includes two classes, such as unoccupied and occupied band. Thus, the size of solution vector is denoted as, $[1 \times(r \times 2)]$, where, $r$ denotes the total number of classes.

\section{b) Fitness function}

The fitness function is based on the minimal objective constraint that is nothing, but the clustering objective. However, the fitness function is expressed as,

$$
F=\sum_{i=1}^{D} \sum_{j=1}^{W}\left(T_{i}-f_{j}\right)
$$

where, $T_{i}$ denotes $i^{\text {th }}$ centroid, and $f_{j}$ represents the data point corresponds to $i^{\text {th }}$ centroid. The algorithmic steps involved in the proposed Adaptive WEHO algorithm are elaborated as follows:

Step 1: Population initialization: Let us initialize the population of whales in the search space as, ..$s_{j} ;(1 \leq j \leq X)$, Here $X$ specifies the total number of whales in the search space.

Step 2: Fitness evaluation: The fitness function is evaluated for all the search agents based on the Eq. (14) such that the best solution is obtained based on the minimal fitness value.

Step 3: Update the position of search agent: The hunting behavior of whales is modeled using three different phases explained below: Encircling is the first phase in the optimization algorithm, where each search agent finds the location of prey individually. Once the location of best search agent is specified then, the remaining search agent in the whale groups updates their location. Hence, the hunting behavior of encircling phase is represented as,

$$
\begin{aligned}
& \vec{Y}=\left|\overrightarrow{R_{1}} \cdot \overrightarrow{s^{*}}(t)-s(t)\right| \\
& s(\overrightarrow{t+1})=\overrightarrow{s^{*}}(t)-\overrightarrow{R_{2}} \cdot \vec{Y}
\end{aligned}
$$

By substituting the Eq. (15) in Eq. (16), the resulted equation is expressed as,

$$
s(\overrightarrow{t+1})=\overrightarrow{s^{*}}(t)-\overrightarrow{R_{2}} \cdot\left|\overrightarrow{R_{1}} \cdot \overrightarrow{s^{*}}(t)-s(t)\right|
$$

Let us assume the term $\left|\overrightarrow{R_{1}} \cdot \overrightarrow{s^{*}}(t)-s(t)\right|$ as positive, hence the above equation is rewritten as,

$$
\begin{aligned}
& s(\overrightarrow{t+1})=\overrightarrow{s^{*}}(t)-\overrightarrow{R_{2}} \cdot\left(\overrightarrow{R_{1}} \cdot \overrightarrow{s^{*}}(t)-s(t)\right) \\
& s(\overrightarrow{t+1})=\overrightarrow{s^{*}}(t)\left[1-\overrightarrow{R_{1}} \cdot \overrightarrow{R_{2}} \cdot\right]+\overrightarrow{R_{2}} \cdot \vec{s}(t)
\end{aligned}
$$

where, $t$ represents present iteration, $\vec{R}_{1}$ and $\vec{R}_{2}$ are the coefficient vectors, $s(t)$ denotes position vector, || indicates the element wise multiplication, and $\vec{s}^{*}(t)$ indicates the position vector of best solution. The coefficient vectors are computed as,

$$
\begin{aligned}
& \overrightarrow{R_{2}}=2 \vec{g} \cdot \vec{w}-\vec{g} \\
& \vec{R}_{1}=2, \vec{w}
\end{aligned}
$$

where, $\vec{g}$ takes the value between $[0,2]$, and $\vec{w}$ denotes the random number that lies between 0 to 1 . Hence, the search agent updates their location based on the distance vector, and position of best search agent. Second phase is the attacking phase known as bubble net attacking phase, which specifies the spiral update and the shrinking encircling mechanism. Based on the search agent updates their position based on the distance of search agent. Hence, the update equation of attacking phase is represented as,

$$
s(\overrightarrow{t+1})=\overrightarrow{Y^{\prime}} \cdot e^{b k} \cdot \cos (2 \pi k)+\vec{s}^{*}(t)
$$

where, $\vec{Y}^{\prime}$ denotes the distance, which is denoted as, $\overrightarrow{Y^{\prime}}=\left|\overrightarrow{s^{*}}(t)-\vec{s}(t)\right|$. Hence the above equation is modified as,

$$
s(t+1)=\left|\overrightarrow{s^{*}}(t)-s \vec{s}(t)\right| \cdot e^{b k} \cdot \cos (2 \pi k)+\overrightarrow{s^{*}}(t)
$$

Let us assume the distance as positive, and hence the above equation is modified as,

$$
\begin{array}{r}
s(\overrightarrow{t+1})=\left(\overrightarrow{s^{*}}(t)-\vec{s}(t)\right) e^{b k} \cdot \cos (2 \pi k)+\overrightarrow{s^{*}}(t) \\
s(\overrightarrow{t+1})=\vec{s}^{*}(t)\left(e^{b k} \cdot \cos (2 \pi k)+1\right)-\vec{s}(\vec{t}) \cdot e^{b k} \cdot \cos (2 \pi k)
\end{array}
$$

where, $k$ denotes the random number that lies between -1 to 1 , and $b$ represents the shape of spiral movement. The probability factor decides the mechanism of spiral model or shrinking encircling phase. Hence, the search agent update the position with the probability represented as,

$$
s(\overrightarrow{t+1})= \begin{cases}\overrightarrow{s^{*}}(t)\left[1-\vec{R}_{2} \vec{R}_{1}\right]+\vec{R}_{2} \cdot \vec{s}(t) & ; \quad \text { if } \beta<0.5 \\ \overrightarrow{s^{*}}(t)\left(e^{b k} \cdot \cos (2 \pi k)+1\right)-s(\vec{t}) \cdot e^{b k} \cdot \cos (2 \pi k) & ; \quad \text { if } \beta \geq 0.5\end{cases}
$$

where, $\beta$ denotes the random number lies between $[0,1]$. The exploration and the exploitation phase are achieved based on $\left|\vec{R}_{2}\right|$, when $\left|\vec{R}_{2}\right|$ is higher than 1 then, the phase is determined as exploitation phase. 
Hence, the spiral model is specified as,

$$
\begin{aligned}
& \vec{Y}=\left|\vec{R}_{1} \cdot \vec{s}_{\text {rand }}-\vec{s}\right| \\
& s\left(\overrightarrow{t+1)}=\vec{s}_{\text {rand }}-\vec{R}_{2} \cdot \vec{Y}\right.
\end{aligned}
$$

where, $S_{\text {rand }}$ denotes the position of search agent. However, the random search is performed when $\left|\vec{R}_{2}\right|>1$ and the optimal solution is selected when $\left|\vec{R}_{2}\right|<1$. The standard equation of EHO algorithm is expressed as,

$$
\begin{aligned}
& s(\overrightarrow{t+1})=\vec{s}(t)+\varphi\left(s^{*}(\vec{t})-s(t)\right) \times d \\
& s(\overrightarrow{t+1})=\vec{s}(t)(1-\varphi \times d)+\varphi \times \vec{s}^{*}(t) \times d
\end{aligned}
$$

By rearranging the above equation,

$$
\vec{s}(t)=\frac{1}{(1-\varphi \times d)}\left[s(t+1)-\varphi \times d \times \vec{s}^{*}(t)\right]
$$

The standard equation of WOA in the encircling phase is modified using the above equation as,

$$
\begin{aligned}
& s(\overrightarrow{t+1})=\vec{s}^{*}(t)\left[1-\vec{R}_{1} \cdot \vec{R}_{2} \cdot\right]+\vec{R}_{2} \cdot\left\{\frac{1}{(1-\varphi \times d)}\left[s(\overrightarrow{t+1})-\varphi \times d \times \vec{s}^{*}(t)\right]\right\} \\
& s(\overrightarrow{t+1})-\vec{R}_{2} \frac{1}{(1-\varphi \times d)} s(\overrightarrow{t+1})=\overrightarrow{s^{*}}(t)\left[1-\vec{R}_{1} \cdot \vec{R}_{2}-\vec{R}_{2} \frac{\varphi \times d}{(1-\varphi \times d)} \cdot\right] \\
& s(\overrightarrow{t+1})\left[1-\vec{R}_{2} \frac{1}{(1-\varphi \times d)}\right]=\overrightarrow{s^{*}}(t)\left[1-\vec{R}_{1} \cdot \vec{R}_{2}-\vec{R}_{2} \frac{\varphi \times d}{(1-\varphi \times d)} \cdot\right] \\
& s(\overrightarrow{t+1})=\frac{1-\varphi \times d}{(1-\varphi \times d)-\vec{R}_{2}}\left\{\overrightarrow{s^{*}}(t)\left[1-\vec{R}_{1} \cdot \vec{R}_{2}-\vec{R}_{2} \frac{\varphi \times d}{(1-\varphi \times d)} \cdot\right]\right\}
\end{aligned}
$$

Moreover, the equation of shrinking phase is updated using the Eq. (31) is represented as,

$$
\begin{aligned}
& s(\overrightarrow{t+1})=\vec{s}^{*}(t)\left(e^{b k} \cdot \cos (2 \pi k)+1\right)-\frac{1}{(1-\varphi \times d)}\left[s(\overrightarrow{t+1})-\varphi \times d \times \vec{s}^{*}(t)\right] \cdot e^{b k} \cdot \cos (2 \pi k) \\
& s(\overrightarrow{t+1})+\frac{e^{b k} \cdot \cos (2 \pi k)}{(1-\varphi \times d)} \times s(\vec{t}+1)=\overrightarrow{s^{*}}(t)\left\{\left(e^{b k} \cdot \cos (2 \pi k)+1\right)+\frac{\varphi \times d}{(1-\varphi \times d)} \cdot e^{b k} \cdot \cos (2 \pi k)\right\} \\
& s(\overrightarrow{t+1})=\frac{(1-\varphi \times d) \times \overrightarrow{s^{*}}(t)}{1-\varphi \times d+e^{b k} \cdot \cos (2 \pi k)}\left\{\left(e^{b k} \cdot \cos (2 \pi k)+1\right)+\frac{\varphi \times d}{(1-\varphi \times d)} \cdot e^{b k} \cdot \cos (2 \pi k)\right\}
\end{aligned}
$$

Therefore, the update equation of the proposed Adaptive WEHO algorithm at the encircling and shrinking phase is expressed as,

$$
s(\overrightarrow{t+1})=\left\{\begin{array}{l}
\frac{1-\varphi \times d}{(1-\varphi \times d)-\vec{R}_{2}}\left\{\overrightarrow{s^{*}}(t)\left[1-\vec{R}_{1} \cdot \overrightarrow{R_{2}}-\vec{R}_{2} \frac{\varphi \times d}{(1-\varphi \times d)} \cdot\right]\right\} \quad ; \quad \text { if } \beta<0.5 \\
\frac{(1-\varphi \times d) \times \vec{s}^{*}(t)}{1-\varphi \times d+e^{b k} \cdot \cos (2 \pi k)}\left\{\left(e^{b k} \cdot \cos (2 \pi k)+1\right)+\frac{\varphi \times d}{(1-\varphi \times d)} \cdot e^{b k} \cdot \cos (2 \pi k)\right\} ; \text { if } \beta \geq 0.5
\end{array}\right.
$$

Here, the adaptive parameter $d$ is represented as,

$$
d=\frac{f(u(o))}{f(u(o))+f(u(o-1))}
$$

where, $f(u(o))$ denotes the fitness of solution at current iteration, and $f(u(o-1))$ represents the fitness of solution at previous iteration.
Step 4: Evaluating feasibility: The fitness of the search agent is evaluated based on the Eq. (14) such that the best solution is accepted as the optimal solution.

Step 5: Termination: The above steps are repeated until the best solution is retrieved.

\section{GMM prediction for testing}

From the intermediate classes, $C=\left\{C_{x_{1}}, \ldots C_{x_{r}}\right\}$, the final class is obtained as the un-occupied or occupied bands. Hence, the GMM predicts the class as,

$$
r=G M M^{f n l}(\beta, \kappa, v)
$$

\section{RESULTS AND DISCUSSION}

The results and discussion of the proposed Adaptive WEHO algorithm is elaborated in this section.

\section{A. Experimental setup}

The implementation of the proposed algorithm is done in the MATLAB tool using three different channel environments.

a) Evaluation metrics

The performance revealed by the proposed algorithm is evaluated using the metrics, like probability of false alarm, and probability of detection.

Probability of detection: It is defined as the ratio of detected spectrum to the number of available spectrum, which is expressed as,

$$
\mathrm{A}=\frac{p}{q}
$$

where, $P$ denotes number of detected spectrum, and $q$ represents the available spectrum, respectively.

Probability of false alarm: It is the defined as the ratio of spectrum detected falsely to the total available bands, which is represented as,

$$
\mathrm{B}=\frac{\sigma}{\tau}
$$

where, $\sigma$ denotes falsely detected spectrum, and $\tau$ is the total bands.

\section{B. Comparative methods}

The performance of the proposed algorithms is revealed by comparing the proposed with the existing methods, like GLRT [17], MMED [17], and MED [17].

\section{Comparative analysis}

The comparative analysis of the proposed Adaptive WEHO algorithm at different channels in the presence of 25 and 50 users are elaborated in this section.

\section{Comparative analysis of Rayleigh channel}

The comparative analysis made using the proposed Adaptive WEHO algorithm with the Rayleigh channel is explained in this section.

a) In the presence of 25 users

Figure 2 shows the comparative analysis of Rayleigh channel with 25 users by varying the gamma.

\section{Published By:}


Figure 2 a) shows the analysis of probability of detection. When gamma $=0.97$, probability of detection obtained by the existing methods, like GLRT is 0.7127, MMED is 0.6182 , MED is 0.7263, WEHO is 0.7700 , while the proposed Adaptive

WEHO obtained the probability of detection as 0.8287 , respectively. Figure 2 b) portrays the analysis of probability of false alarm. When gamma $=0.97$, probability of false alarm obtained by the existing methods, like GLRT is 0.781, MMED is 0.600, MED is 0.533, WEHO is 0.456, while the proposed Adaptive WEHO obtained the probability of false alarm as 0.403 , respectively.

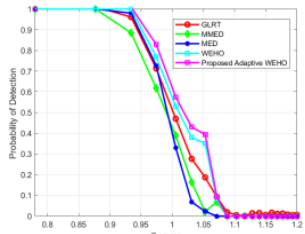

(a)

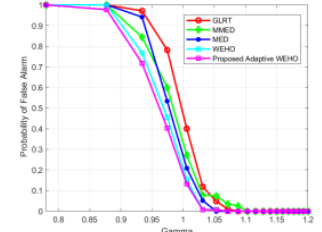

(b)
Figure 2. Comparative analysis of Rayleigh channel with 25 users, a) probability of detection, b) probability of false alarm

b) In the presence of 50 users

Figure 3 shows the comparative analysis of Rayleigh channel with 50 users by varying the gamma. Figure 3 a) shows the analysis of probability of detection. When gamma $=0.97$, probability of detection obtained by the existing methods, like GLRT is 0.7601, MMED is 0.6809 , MED is 0.7308 , WEHO is 0.8200 , while the proposed Adaptive WEHO obtained the probability of detection as 0.9295, respectively. Figure $3 \mathrm{~b}$ ) represents the analysis of probability of false alarm. When gamma $=0.97$, probability of false alarm obtained by the existing methods, like GLRT is 0.9082, MMED is 0.5755 , MED is 0.5313, WEHO is 0.4880 , while the proposed Adaptive WEHO obtained the probability of false alarm as 0.4497 , respectively.

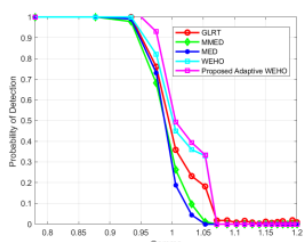

(a)

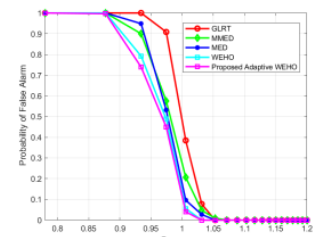

(b)
Figure 3. Comparative analysis of Rayleigh channel with 50 users, a) probability of detection, b) probability of false alarm

\section{Comparative analysis of Rician channel}

The comparative analysis made using the proposed Adaptive WEHO algorithm with the Rician channel is explained in this section.

a) In the presence of 25 users

Figure 4 shows the comparative analysis of Rician channel with 25 users by varying the gamma. Figure 4 a) shows the analysis of probability of detection. When gamma $=0.97$, probability of detection obtained by the existing methods, like GLRT is 0.0.849, MMED is 0.774,
MED is 0.846, WEHO is 0.910, while the proposed Adaptive WEHO obtained the probability of detection as 0.963, respectively. Figure $4 \mathrm{~b}$ ) depicts the analysis of false alarm. When gamma $=0.97$, probability of false alarm obtained by the existing methods, like GLRT is 0.813, MMED is 0.523, MED is 0.594 , WEHO is 0.472 , while the proposed Adaptive WEHO obtained the probability of false alarm as 0.414 , respectively.

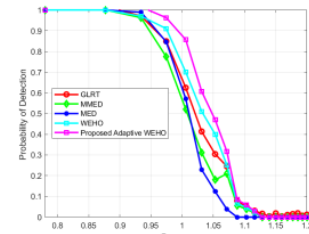

(a)

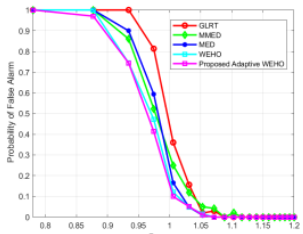

(b)
Figure 4. Comparative analysis of Rician channel with 25 users, a) probability of detection, b) probability of false alarm

b) In the presence of 50 users

Figure 5 shows the comparative analysis of Rician channel with 50 users by varying the gamma. Figure 5 a) portrays the analysis of probability of detection. When gamma $=0.97$, probability of detection obtained by the existing methods, like GLRT is 0.913, MMED is 0.832, MED is 0.990, WEHO is 0.990, while the proposed Adaptive WEHO obtained the probability of detection as 1.135, respectively. Figure 5 b) depicts the analysis of false alarm. When gamma $=0.97$, probability of false alarm obtained by the existing methods, like GLRT is 0.924, MMED is 0.602, MED is 0.556, WEHO is 0.504, while the proposed Adaptive WEHO obtained the probability of false alarm as 0.459 , respectively.

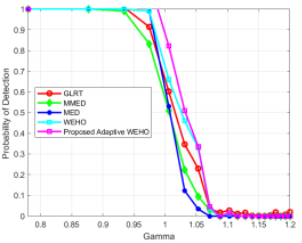

(a)

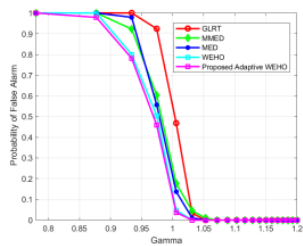

(b)
Figure 5. Comparative analysis of Rician channel with 50 users, a) probability of detection, b) probability of false alarm

\section{Comparative analysis of Gaussian channel}

The comparative analysis made using the proposed Adaptive WEHO algorithm with the Gaussian channel is explained in this section.

a) In the presence of 25 users

Figure 6 shows the comparative analysis of Gaussian channel with 25 users by varying the gamma. Figure 6 a) shows the analysis of probability of detection. When gamma $=0.97$, probability of detection obtained by the existing methods, like GLRT is 0.809, MMED is 0.688, MED is 0.890, WEHO is 0.920, while the proposed Adaptive WEHO obtained the probability of detection as 0.932, respectively. Figure 6 b) depicts the analysis of probability of false alarm. When gamma 0.97 , probability of false alarm obtained by the existing methods, like GLRT is 0.802, MMED is 0.471, MED is 0.590 , WEHO is 0.480 , while the proposed Adaptive WEHO obtained the probability of false alarm as 0.424 , respectively. 


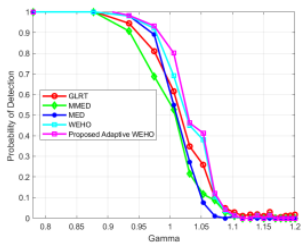

(a)

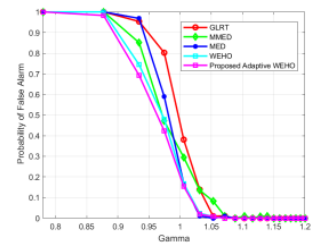

(b)
Figure 6. Comparative analysis of Gaussian channel with 25 users, a) probability of detection, b) probability of false alarm

\section{b) In the presence of 50 users}

Figure 7 shows the comparative analysis of Gaussian channel with 50 users by varying the gamma. Figure 7 a) shows the analysis of probability of detection. When gamma $=0.97$, probability of detection obtained by the existing methods, like GLRT is 0.894, MMED is 0.820, MED is 0.941, WEHO is 0.930, while the proposed Adaptive WEHO obtained the probability of detection as 1.001, respectively. Figure $7 \mathrm{~b}$ ) represents the analysis of false alarm. When gamma $=0.97$, probability of false alarm obtained by the existing methods, like GLRT is 0.910, MMED is 0.550, MED is 0.531, WEHO is 0.504, while the proposed Adaptive WEHO obtained the probability of false alarm as 0.512 , respectively.

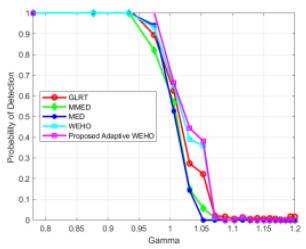

(a) (b)

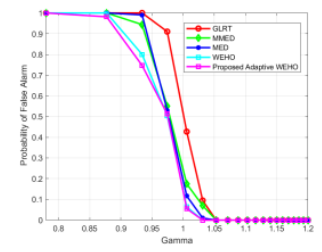

Figure 7. Comparative analysis of Gaussian channel with 50 users, a) probability of detection, b) probability of false alarm

\section{CONCLUSION}

In this research, the spectrum sensing is performed in the MIMO system using the proposed Adaptive WEHO algorithm. Initially, the signals are received from the OFDM receiver and are subjected to the Eigen statistics and signal energy to predict the availability of spectrum in CR network. The proposed hybrid mixture model is done using Gaussian Mixture Model. The GMM parameters are tuned by the proposed Adaptive WEHO algorithm, which is the integration of the EHO and WOA with the adaptive concept. The proposed Adaptive WEHO incorporates the parametric features of hunting and the foraging behavior of whales to ensure the effectiveness of spectral efficiency. The proposed hybrid mixture model revealed better throughput and higher convergence by the characteristic features of humpback whales. The update equation of EHO is modified with the update standard equation of WOA using adaptive concept to achieve the proposed hybrid model. The foraging and the herding behavior of the proposed Adaptive WEHO achieved efficient spectrum sensing in the MIMO system. However, the performance revealed by the proposed algorithm is specified with the lower false alarm probability and higher detection probability of 0.01075 , and 1.0238, respectively. The proposed Adaptive WEHO optimally tunes the Gaussian parameters, which results in a better performance of prediction. Moreover, the proposed hybrid mixture model derives the spectrum availability and ensures the effective communication in CR without any interference.

\section{REFERENCES}

1. Thangaraj, C.A. and Aruna, T., "Energy-Efficient Power Allocation with Guaranteed QoS Under Imperfect Sensing for OFDM-Based Heterogeneous Cognitive Radio Networks", Wireless Personal Communications, pp.1-18, 2019.

2. Viswanath, V., Alam, S. and Kshetrimayum, R.S., "Spectrum Sensing and Collision with Primary Users in MIMO Cognitive Radio", IEEE Twenty Fourth National Conference on Communications (NCC), pp. 1-6, February 2018.

3. Patel, A., Ram, H., Jagannatham, A.K. and Varshney, P.K., "Robust cooperative spectrum sensing for MIMO cognitive radio networks under CSI uncertainty", IEEE Transactions on Signal Processing, vol. 66, no. 1, pp.18-33, 2017.

4. Ren, X. and Chen, C., "Spectrum sensing algorithm based on sample variance in multi-antenna cognitive radio systems", AEU-International Journal of Electronics and Communications, vol. 70, no. 12, pp.16011609, 2016.

5. Wang, G.G., Deb, S. and Coelho, L.D.S., "Elephant herding optimization", IEEE 3rd International Symposium on Computational and Business Intelligence (ISCBI), pp. 1-5, December 2015.

6. Seyedali Mirjalili and Andrew Lewis, "The Whale Optimization Algorithm," Advances in Engineering Software vol.95, pp.51-67, 2016.

7. Mitola, J. and Maguire, G.Q., "Cognitive radio: making software radios more personal”, IEEE personal communications, vol. 6, no. 4, pp.13-18, 1999.

8. Haykin, S., "Cognitive radio: brain-empowered wireless communications", IEEE journal on selected areas in communications, vol. 23, no. 2, pp.201-220, 2005.

9. Zhu, X., Shen, L. and Yum, T.S.P., "Analysis of cognitive radio spectrum access with optimal channel reservation", IEEE Communications Letters, vol. 11, no. 4, pp. 304-306, 2007.

10. Subhedar, M. and Birajdar, G., "Spectrum sensing techniques in cognitive radio networks: A survey", International Journal of NextGeneration Networks, vol. 3, no. 2, pp.37-51, 2011.

11. Pandit, S. and Singh, G.,. "Spectrum sensing in cognitive radio networks: potential challenges and future perspective", In Spectrum Sharing in Cognitive Radio Networks, pp. 35-75, Springer, Cham. 2017

12. Suliman, I., Lehtomäki, J., Bräysy, T. and Umebayashi, K., "Analysis of cognitive radio networks with imperfect sensing", IEEE 20th International Symposium on Personal, Indoor and Mobile Radio Communications, pp. 1616-1620, September 2009.

13. Altrad, O., Muhaidat, S., Al-Dweik, A., Shami, A. and Yoo, P.D., "Opportunistic spectrum access in cognitive radio networks under imperfect spectrum sensing", IEEE Transactions on Vehicular Technology, vol. 63, no. 2, pp.920-925, 2013.

14. Urkowitz, H., "Energy detection of unknown deterministic signals", Proceedings of the IEEE, vol. 55, no. 4, pp.523-531, 1967.

15. Chaudhari, S., Koivunen, V. and Poor, H.V., "Autocorrelation-based decentralized sequential detection of OFDM signals in cognitive radios", IEEE transactions on signal processing, vol. 57, no. 7, pp.2690-2700, 2009.

16. XinxinXie, Wenzhun Huang, Harry Haoxiang Wang, and Zhe Liu , "Image De-noising Algorithm based on Gaussian Mixture Model and Adaptive Threshold Modeling", In Proceedings on the International Conference on Inventive Computing and Informatics (ICICI), 2017.

17. Dayan A. Guimaraes, Carlos R. N. da Silva, and Rausley A. A. de Souza, "Cooperative Spectrum Sensing Using Eigenvalue Fusion for OFDMA and Other Wideband Signals", Journal of Sensor and Actuator Networks, 2013. 